\title{
Fistule artério-urétérale après une pelvectomie antérieure : Complication rare et grave
}

\author{
Mohamed Hosni Zine el abidine, MD ; Wissem Hmida, MD ; Adel Slama, MD ; Adnene Hidoussi, MD ; \\ Mehdi Jaidane, MD ; Nabil Ben Sorba, MD ; Ali Fawzi Mosbah, MD
}

Service d'Urologie, CHU Sahloul, Sousse, Tunisie

Cite as: Can Urol Assoc J 2012;6(6):E263-E266. http://dx.doi.org/10.5489/cuaj.11157

\section{Résumé}

La fistule artério-urétérale (FAU), qui survient immédiatement après une cystectomie, est une complication exceptionnelle et grave. De nos jours, seulement quelques cas sporadiques ont été signalés dans la littérature. Elle se manifeste presque exclusivement par une hématurie brutale, massive et intermittente pouvant être à l'origine d'une instabilité hémodynamique menaçant le pronostic vital du patient à court terme. Son diagnostic doit être fait sans retard. Les explorations radiologiques restent peu spécifiques et peu sensibles et se basent essentiellement sur l'artériographie. Le traitement est non codifié selon l'état général du patient et surtout de la nature de la fistule. Nous décrivons un cas de FAU faisant suite à une pelvectomie antérieure et nous discuterons à l'aide d'une revue de la littérature les aspects diagnostiques, thérapeutiques et pronostiques de cette complication.

\section{Introduction}

La fistule artério-urétérale (FAU) est une complication très rarement décrite dans les suites immédiates d'une pelvectomie antérieure ${ }^{1,2}$. Il s'agit d'une complication grave pouvant mettre en jeu à court terme le pronostic vital du patient par le saignement important qu'elle va engendrer ${ }^{3,4}$. Son diagnostic est souvent difficile, et parfois déroutant et tardif. Il doit être établi sans retard sur des arguments cliniques et radiologiques. L'hématurie massive et intermittente reste le maître symptôme. Les explorations radiologiques sont peu spécifiques et peu sensibles; elles se basent essentiellement sur les données de l'artériographie. Le traitement est non codifié selon l'état général du patient et surtout la nature de la fistule.

Nous signalons un nouveau cas de FAU survenue immédiatement après une pelvectomie antérieure avec dérivation urinaire externe de type Bricker pour traiter une tumeur vésicale infiltrante. Nous discuterons à l'aide d'une revue de la littérature les aspects diagnostiques, thérapeutiques et pronostiques de cette complication.

\section{Observation}

Madame Z. K., 60 ans, obèse (indice de masse corporelle de 35) et ne présentant pas d'antécédents pathologiques notables, notamment vasculaires, a été hospitalisée pour prise en charge d'une tumeur vésicale infiltrante. Une TDM abdominopelvienne préopératoire avait permis de conclure à la présence d'une tumeur vésicale T3NOMO. La patiente a subi une pelvectomie antérieure avec curage ganglionnaire ilio-obturateur bilatéral et une urétrectomie totale. Une dérivation urinaire externe trans-iléale de type Bricker a été effectuée. Le geste opératoire s'est déroulé sans incident ni complication peropératoire, notamment au moment du curage ganglionnaire, de la dissection des deux uretères et de l'anastomose urétéro-iléale. Les deux uretères étaient drainés par deux sondes urétérales.

Les suites postopératoires immédiates étaient marquées au deuxième jour par un état septique sévère; les hémocultures ont permis d'isoler un type multirésistant d'Escherichia coli. La patiente a été mise sous double antibiothérapie bien adaptée. Au $10^{\mathrm{e}}$ jour après l'opération et lors de l'ablation des sondes urétérales, elle a présenté une hématurie de grande abondance, intermittente, à l'origine d'une instabilité hémodynamique et nécessitant des transfusions itératives.

Dans I'enquête étiologique, une échographie abdominopelvienne montrait un rein gauche atteint $d^{\prime}$ une dilatation urétéro-pyélocalicielle (DUPC) modérée avec un contenu finement échogène sans obstacle identifiable. L'examen Doppler amenait à soupçonner une thrombose partielle de la veine rénale gauche sans atteinte de la veine cave.

Un examen urologique par IRM a montré une importante DUPC gauche à contenu hétérogène avec des niveaux liquide-liquide attestant une nature hémorragique sans visualisation de processus tumoral urothélial patent, et confirmait la thrombose partielle de la veine rénale gauche. 


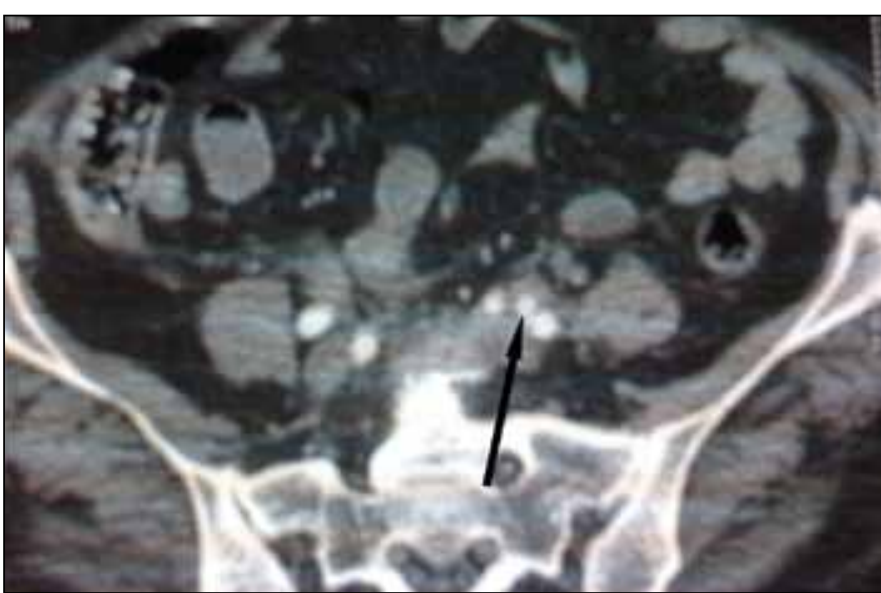

Fig.1a. Angiographie (coupe axiale) : opacification de l'uretère (flèche).

Vu l'importance, la persistance et l'aspect artériel du saignement, on a alors soupçonné la présence d'une fistule artério-urétérale; le diagnostic a été confirmé par angiographie (Fig. 1a, Fig. 1b).

Une exploration chirurgicale urgente était alors indiquée et a mis en évidence une fistule faisant communiquer l'artère iliaque externe et la portion iliaque de l'uretère gauche. La paroi artérielle était fragilisée. On a procédé à la suture de I'orifice fistuleux avec patch (Fig. 2a, Fig. 2b), et on a opté pour une néphro-urétérectomie.

Les suites opératoires étaient simples avec disparition de I'hématurie et stabilisation du profil hémodynamique. Le membre inférieur gauche était sans anomalie à l'examen clinique et bien vascularisé à l'examen Doppler.

\section{Discussion}

La fistule artério-urétérale après une chirurgie pelvienne reste une entité anatomoclinique rare. Jusqu'à présent, seuls quelques cas sporadiques ont été signalés dans littérature ${ }^{1}$. Il s'agit d'une complication grave pouvant menacer le pronostic vital, surtout si elle est diagnostiquée tardivement ${ }^{3}$. La quasi-majorité des FAU sont secondaires (85\%).

Plusieurs étiologies ont été signalées ${ }^{1,3,5-7}$ entre autres :

La chirurgie génito-urinaire ou pelvienne (68\%), notamment la chirurgie carcinologique, est la cause la plus fréquente ${ }^{8,9}$. Il s'agit surtout de la chirurgie d'exérèse des tumeurs localement avancées, comme c'était le cas pour notre patiente.

- Le cathétérisme urétéral prolongé vient en deuxième position, il est en cause dans $60 \%$ des $\operatorname{cas}^{5,10}$. Les FAU surviennent alors surtout lors de l'utilisation des sondes rigides et de gros calibre².

- La radiothérapie abdominopelvienne (46\% des cas) : la FAU est due probablement à une dévascularisation urétérale et une nécrose tissulaire localisée ${ }^{3,10}$.

- La chirurgie vasculaire aorto-iliaque (19\%) : dans ce

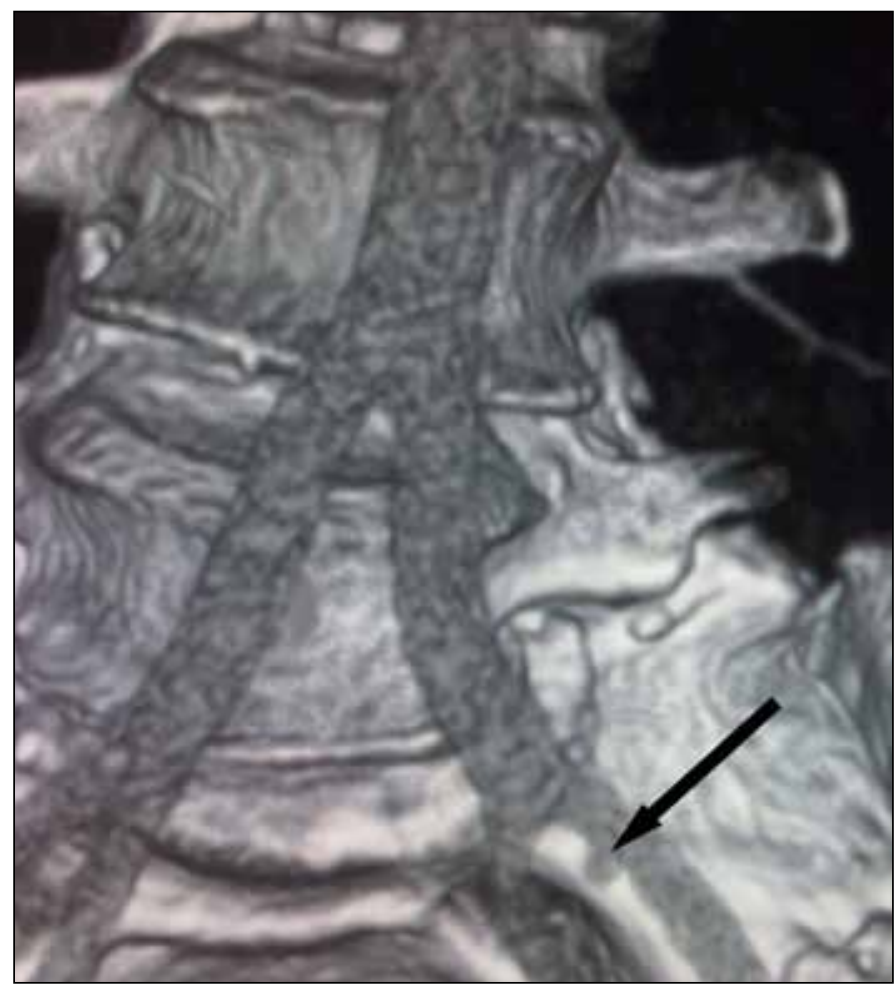

Fig.1b. Angiographie (coupe sagittale) montrant le trajet de la FAU (flèche).

cas, la FAU survient par exemple lors de la mise en place d'une prothèse $\mathrm{d}^{2,3,9}$.

- L'infection constitue aussi un facteur de risque de FAU, que ce soit des infections urinaires telles que pyélonéphrites, pyonéphroses et urinomes infectés ${ }^{11-13}$, ou des infections vasculaires, comme l'infection d'une prothèse $\mathrm{e}^{12,14}$.

- Enfin I'origine traumatique reste exceptionnelle. Un seul cas de FAU par suite d'une blessure par arme à feu a été signalé dans la littérature ${ }^{11}$.

Par ailleurs, les FAU peuvent être primitives (15\% des cas), survenant essentiellement sur un terrain de pathologie vasculaire artérielle, surtout un anévrysme aorto-iliaque'.

Le mécanisme physiopathologique des FAU reste encore mal élucidé; il implique plusieurs facteurs intriqués. L'hypothèse la plus plausible est celle de la transmission constante des battements artériels de l'axe iliaque à la paroi urétérale, fragilisée par une dissection chirurgicale, une radiothérapie ou infection, et entraînant donc une nécrose de la zone de contact, aggravée, bien entendu, par un cathétérisme urétéral ${ }^{15,16}$. Ce dernier tamponne temporairement la fistule, ce qui explique en grande partie l'intermittence de I'hématurie et son caractère cataclysmique lors de l'ablation de la sonde ${ }^{17}$. L'intermittence de l'hématurie s'explique aussi par la formation de caillots sanguins au point d'origine de la fistule ${ }^{2}$.

Sur le plan clinique, la FAU se manifeste presque exclusivement par une hématurie brutale, massive et intermit- 
Fistule artério-urétérale après une pelvectomie antérieure

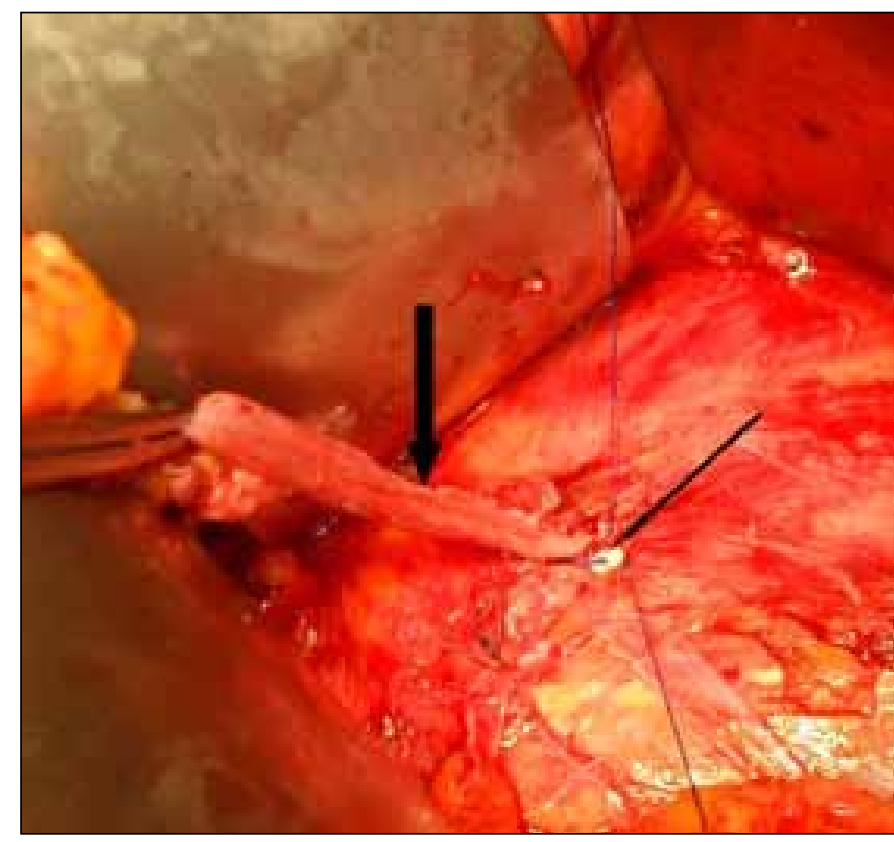

Fig.2a. *Flèche épaisse : uretère ; ${ }^{*}$ Flèche mince : siège de la FAU et début de la suture sur patch.

tente pouvant être à l'origine d'une instabilité hémodynamique $^{4,5,10-14,18}$. Cette hématurie peut $\mathrm{s}^{\prime}$ associer parfois à des douleurs lombaires secondaires à une dilatation des voies excrétrices en amont d'un caillot sanguin urétéral, comme c'était le cas avec notre patiente.

Holmes et al. ont décrit une triade clinique associant une hématurie intermittente, une hydronéphrose et des antécédents de chirurgie vasculaire iliaque ${ }^{4}$.

Les examens complémentaires sont peu spécifiques et peu sensibles si le diagnostic de FAU n'a pas été évoqué auparavant. Ils permettent par ailleurs d'éliminer les autres causes d'hématurie ${ }^{1,6,7,14,17}$.

De nos jours, I'UIV est supplantée par la TDM abdominopelvienne, qui est le premier examen demandé. Néanmoins, elle reste peu contributive pour le diagnostic positif, et son rôle réside dans l'évaluation de la fonction rénale et la vérification d'une dilatation urétéro-pyélocalicielle. Elle permet aussi d'éliminer une tumeur du rein ou de la voie excrétrice comme source de l'hématurie. L'UIV peut aussi montrer un rapport étroit entre l'uretère et l'axe artériel sans mettre en évidence le trajet fistuleux $x^{4,5,17}$.

L'artériographie est peu sensible, elle confirme le diagnostic dans seulement $30 \%$ des cas. La performance de cet examen est améliorée par une manœuvre de provocation du cathétérisme urétéral, le cas échéant. Sa sensibilité est nettement améliorée et peut ainsi atteindre $100 \% 19,20$. Effectuée par un expert, l'artériographie permet en plus un geste endovasculaire d'hémostase ${ }^{19}$.

L'exploration endoscopique peut être utile dans certains cas en montrant l'origine du saignement. Néanmoins, ceci $n^{\prime}$ est possible que si le saignement est minime ${ }^{11,12}$.

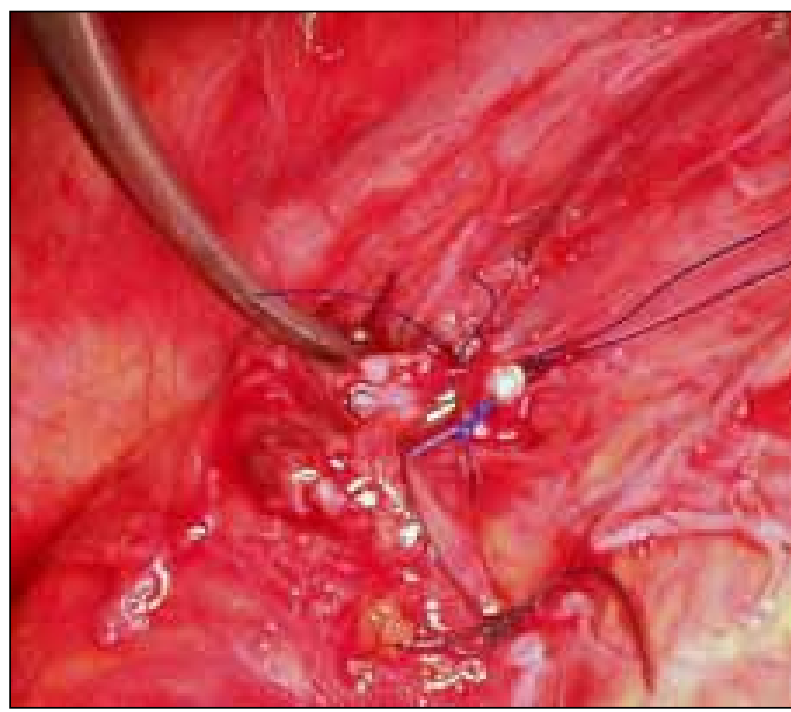

Fig. $2 \boldsymbol{b}$. Pointe de la pince : fin de la suture sur patch.

L'urétéropyélographie descendante ou au mieux rétrograde est l'examen de référence qui permet de visualiser un passage artériel du produit de contraste dans $60 \%$ des cas $^{10,19,21}$. Toutefois, cet examen doit être réalisé avec prudence, puisqu'une mobilisation du caillot peut amener une reperméabilisation du trajet fistuleux, ce qui déclencherait une hématurie cataclysmique $e^{5,10,15,18}$.

Enfin, et dans plusieurs cas signalés dans littérature, le diagnostic est posé lors d'une exploration chirurgicale faite le plus souvent dans un contexte $d^{\prime}$ urgence ${ }^{2,11,12}$.

Le traitement des FAU comporte deux étapes : une étape vasculaire d'hémostase et une étape urologique.

En effet, plusieurs options sont offertes pour le traitement de la lésion artérielle :

- suture directe de l'orifice fistuleux avec ou sans patch ;

- mise à plat de la fistule et reconstitution par un pontage ilio-fémoral ;

- ligature artérielle, s'il s'agit de l'artère iliaque interne.

La complication la plus redoutée lors de la réparation artérielle est la rupture iliaque secondaire ${ }^{22}$.

Le traitement endovasculaire (embolisation, endoprothèse) a connu récemment un essor important et il est actuellement de plus en plus utilisée ${ }^{16,20}$. Toutefois, l'évolution à long terme est mal connue et expose à un risque accru de thrombose, de récidive et surtout d'infection ${ }^{3,6}$.

Le traitement urologique, deuxième volet de la prise en charge, n'est pas codifié et comporte plusieurs possibilités :

- néphro-urétérectomie ;

- ligature urétérale avec néphrostomie percutanée ;

- résection anastomose termino-terminale urétérale ;

- réimplantation urétéro-vésicale.

Le choix dépendra surtout de l'état général du patient et de la nature de la fistule.

Le pronostic des FAU s'est nettement amélioré ces der- 
nières années grâce aux progrès des techniques diagnostiques et thérapeutiques ${ }^{6,7,23}$. En effet, la mortalité en lien avec les FAU est passée de $70 \%$ dans les années 80 à moins de $30 \%$.

La prévention des FAU consisterait en une diminution de la durée du drainage trans-urétéral ainsi que l'utilisation de sondes souples et de faible calibre ${ }^{5,10,17}$. Certains auteurs préconisent une dérivation trans-iléale en position haute, pour éviter le contact de I'uretère avec l'artère iliaque ${ }^{24}$. D'autres, comme Smith et al., conseillent de les espacer par de l'épiploon ${ }^{25}$.

\section{Conclusion}

La FAU survenant immédiatement après une pelvectomie antérieure est une complication rare et redoutable. Elle peut menacer le pronostic vital du patient si le diagnostic se fait tardivement. Il faut toujours envisager cette possibilité, surtout quand l'intervention chirurgicale s'accompagne de difficultés, est compliquée par une infection ou associée à un cathétérisme urétéral. Il faut l'envisager sans délai devant une hématurie massive et intermittente ne répondant pas aux transfusions itératives. Les explorations radiologiques sont peu contributives. Leur normalité peut être considérée comme un élément en faveur du diagnostic. L'exploration chirurgicale urgente, au mieux conservatrice, est souvent nécessaire. À l'heure actuelle, les techniques endovasculaires semblent donner de bons résultats et restent prometteuses.

Competing interests: None declared.

This paper has been peer-reviewed.

\section{Références}

1. Bergqvist D, Pärssson H, Sherif A. Arterio-fistula - a systematic review. Eur J Vasc Endovasc Surg 2001;22:191-6. http://dx.doi.org/10.1053/ejvs.2001.1432

2. Demailly $M$, Gastaud 0 , Hakami F, et al. À propos de deux cas de fistule artério-urétérale. Prog Urol 2001:11:528-33.
3. Lefebure F, de Wailly GW, Chiche L. Fistules urétéro-artérielles : prise en charge diagnostique et thérapeutique. Sang Thrombose Vaisseaux 2006;18:548-54.

4. Holmes $M$, Hung $N$, Hunter $M$. Hematuria and death secondary to aortoureteric fistula. Urology 1998:52:720-2. http://dx.doi.org/10.1016/S0090-4295(98)00281-7

5. Menasse F, Rossi D, Heckenroth H. Fistules urétéro-artérielles: à propos de deux cas et revue de la littérature. Prog Urol 1997;7:1007-11.

6. Dalmas $P$, Haddad 0 , Jacquier $A$, et al. Fistule artério-urétérale : approche diagnostique et thérapeutique. Prog Urol 2001;11:1277-88.

7. Amahzoune $M$, Hilal $R$, Gouillat $C$, et al. Fistule artériourétérale, à propos de trois cas. Arterio-ureteral fistula, report of three cases. Ann de Chir 2005;130:257-60. http://dx.doi.org/10.1016/i. anchir.2005.01.007

8. Toolin E, Pollack HM, McLean GK, et al. Uretero-arterial fistula : a case report. J Urol 1984;132:553-4.

9. Whitmore WF. Uretero-arterial fistula and uretero-vaginal fistula: report of a case. Urologia 1954;21:184.

10. Batter SJ, McGovern FJ, Cambira RP. Ureteroarterial fistula : case report and review of the literature. Urology 1996;48:481-9. http://dx.doi.org/10.1016/50090-4295(96)00202-6

11. Bodak A, Levot $\mathrm{E}$, Schut $\mathrm{A}$, et al. Un cas de fistule artério-urétérale. Revue de la littérature. J Urol 1990;96:55-9.

12. Dauplat J, Piollet H, Condat P, et al. Deux cas de fistules urétéro-artérielles. J Urol (Paris) 1985;91:457-61.

13. Vinee $P$, Hauenstein $K H$, Nökdge $G$, et al. La fistule urétéro-artérielle. Une cause rare d'hématurie massive. $J$ Urol (Paris) 1992;98:165-6.

14. Simon $G$, Ballanger $P$, Midy $D$, et al. Fistules artérielles ilio-urétérales après chirurgie reconstructrice aorto-liaque. Prog Urol 1992;2:85-92.

15. Rodriguez HE, Eggener SE, Podbielski $\mathrm{FJ}$, et al. Occlusion of an intraluminal endovascular stent graft after treatment of ureteral-iliac artery fistula. Urology 2002;60:912. http://dx.doi.org/10.1016/ S0090-4295(02)01902-7

16. Meester DJ, Muiswinkel KW, Ameye F, et al. Endovascular treatment of a uretero-iliac fistula associated with ureteral double $\mathrm{J}$-stent and an aortic-bifemoral stent graft for inflammatory abdominal aortic aneurysm. Ann Vasc Surg 2006;20:408-10.

17. Van Damme H, Keppenne V, Sakalihasan N, et al. Uretero-arterial fistula : two observations. Acta Chir Belg 1997;97:133-6.

18. Andreasen J, Fahrenkrug L, Madsen P. Massive hematuria due to iliac artery-ureteral fistula case report. Eur J Surg 1991;157:223-4.

19. Vandersteen DR, Saxon RR, Fuchs $E$, et al. Diagnosis and management of ureteroiliac fistula : value of provocative arteriography followed by common iliac embolization and extraanatomic arterial by pas $\mathrm{s}$ grafting. J Urol 1997;158:754-8.

20. Kerns DB, Darcy MD, Baumann DS, et al. Autologous vein-covered stent for the endovascular management of an iliac artery-ureteral fistula: case report and review of the literature. J Vasc Surg 1996;24:680-6.

21. Krambeck AE, DiMarco DS, Gettman MT, et al. Ureteroiliac artery fistula : diagnosis and treatment algorithm. Urology 2005;66:990-4. http://dx.doi.org/10.1016/j.urology.2005.05.036

22. Albani JM, Angermeier KW. Right common liac artery pseudoeurysm-uretric fistula. BJU Int 2003;91:890. http://dx.doi.org/10.1046/j.1464-410X.2003.04196.x

23. Fever $D$, Ciocca R, Nackman $G$. Endovascular management of ureteroarterial fistula, case reports. J Vasc Surg 1999;30:1146-9.

24. Puppo P, Perachino M, Ricciotti G, et al. Ureteroarterial fistula : a case report. J Urol 1992; 148:863-4.

25. Smith RB. Ureteral common iliac artery fistula : a complication of internal double J ureteral stenting. J Urol 1984;132:113.

Correspondence: Dr. Mohamed Hosni Zine El Abidine, C. H. U. Sahloul Route Ceinture Cité Sahloul 4054 Sousse, Tunisie ; hosni_zine@hotmail.fr 